\title{
Temporal Analysis of Maternal Mortality in Kano State, Northern Nigeria: A Six-Year Review
}

\author{
YAR'ZEVER S. Ibrahim* \\ Department of Sociology, Bayero University, Kano, Nigeria
}

*Corresponding author: dr.ibyar@yahoo.com

Received September 23, 2013; Revised November 21, 2013; Accepted April 08, 2014

\begin{abstract}
To determine the incidence of maternal mortality associated with non utilization of obstetric services and how socio-demographic and medical factors influence the deaths of pregnant women. A retrospective study of information extracted from hospital records on live birth, antenatal attendance, age at death, parity, educational level and cause of death was reviewed, the study covered data from thirty General hospitals in both urban and rural areas in Kano state for a period of 6 years from $1^{\text {st }}$ January 2005 to $31^{\text {st }}$ December 2010. Data collected was analyzed for pattern of antenatal care, hospital live delivery, age at death, obstetric causes of death and maternal mortality ratio. There were 2,310,727 antenatal attendance 1,886,932 live deliveries and 3,062 maternal death during the period under review given a maternal mortality ratio (MMR) of 1625/100,000 live deliveries. It was also discovered that mean age at death for pregnant women for urban areas was 28.8 years and rural 27.6 years. Both antenatal and hospital live deliveries had irregular pattern. The major direct causes of death in urban area was eclampsia and bleeding cases while in rural areas bleeding cases followed by eclampsia and obstructed labor and then anemia were more prevalent.
\end{abstract}

Keywords: temporal, analysis, maternal mortality, Kano

Cite This Article: YAR'ZEVER S. Ibrahim, “Temporal Analysis of Maternal Mortality in Kano State, Northern Nigeria: A Six-Year Review.” American Journal of Public Health Research, vol. 2, no. 2 (2014): 62-67. doi: 10.12691/ajphr-2-2-5.

\section{Introduction}

Maternal health has emerged as the most important issue that determines global and national wellbeing. This is because every individual, family and community is at some point intimately involved in pregnancy and the success of child birth [1]. Despite the honor bestowed on womanhood and the appreciation of the birth of a new born baby, pregnancy and child birth still considered a perilous journey. Several measures and conferences have been held by the international community to address the problem of maternal death; among others are the United Nations decades for women population conference held in Mexico City in 1984, then the safe motherhood initiative launched in Nairobi, Kenya in 1987, the United Nations Conference on population and development held in Cairo, 1994, the Beijing Conference for women in 1995 and finally the United Nations Millennium Development Goals (MDG'S) in 2000 were all held in an attempt to tackle maternal mortality issues and drew unprecedented attention to reproductive health and rights as well as gender equity and equality. In addition, the Maputo Declaration and Action plan also call for concerted action to reduce maternal mortality, promote maternal health and empower women with knowledge so that they are more useful to themselves, their families and communities.

The situation of maternal health in Nigeria is among the worst in Africa and has not improved substantially and in some areas of the country, has worsened over the years [2] The maternal mortality ratio ranges between $800-1,800$ per 100,000 live births in Nigeria [3] with marked variation between geo-political zones- 165 in south west compared with 1,749 in the North- east and between urban and rural areas [4] while total fertility rate is 5.7 births per woman. It is also estimated that approximately 60,000 of maternal deaths take place annually in Nigeria as a result of pregnancy, delivery and post delivery complications $[5,6]$. Nigeria is second to India in terms of absolute number of maternal deaths and regrettably, despite abundant resources, contributes more than $10 \%$ of all global maternal deaths. The northern part of the county has generally worse indicators [3,7].

Despite the wide range of maternal health services available, maternal mortality in Nigeria continues to rise in some regions. This is not unconnected with the weak management and implementation of health policies and services compounded with the socio-economic and cultural factors. In reducing maternal mortality in Nigeria, the federal government introduced some strategies like free antenatal care for all pregnant women, skilled care delivery during childbirth, post partum family planning counseling and services, training of community midwives to bridge the gap in the rural areas, advocate effective referral system right from primary to tertiary level health care and the recent pre health insurance scheme for all pregnant women lunched by the Federal Government on 3rd February 2011 in Abuja however, years after 
instituting most of these programs, Nigerian women still face the same factors operating at community health care levels in the rural as well as urban areas that increase maternal mortality. It is for these reasons that this study examined the determinants of maternal mortality and the solutions that may be recommended towards improving the health of mothers in both rural and urban areas.

The objectives of the study were:

i). To determine the incidence of maternal mortality associated with non utilization of obstetric services

ii). To determine how socio-demographic and medical factors can influence deaths.

iii). To determine the magnitude and trends in maternal mortality ratio in Kano State.

\section{Methodology}

\subsection{Study Design}

This is a retrospective study conducted for hospital data collected between $1^{\text {st }}$ January 2005 to $31^{\text {st }}$ December 2010 on information on birth, antenatal attendance, age at death, parity, hospital delivery, educational level and causes of maternal mortality.

\subsection{The Study Area}

Kano city is an ancient city with over 1500 years of history [8]. It remains one of the oldest Hausa city-states that enjoy the eminent position of being a foremost centre of commerce, Islamic thought and culture. It is currently the second most populous state in Nigeria after Lagos according to the 2006 census with 10,810,340 peoples of which $51 \%(5,958,736)$ are male and $49 \%(5,851,734)$ are females ${ }^{4}$. The culture of people is Hausa-Islamic culture, in that ethnicity and religion are so interwoven that a distinction is hardly discernable. The practice of polygamy is very common, so are large families and majority of women prefer home delivery. The metropolis is where majority of people with western education resides also where most of the tertiary hospitals are located and a centre of commerce also the site of government.

The love for children and ability to have them is an important component of the social life of the Hausa people making majority of men marrying more than one wife. Therefore, the ability of a woman to bear children influences her social status in the society. It is similar for the male. Women who could not conceive or bear children are stigmatized and labeled as Juya meaning a woman who cannot have a child of her own, and have to adopt. The number of children a woman can bear is an indication of her fertility and social status and standing9.

\subsection{Sampling}

A six year retrospective data from thirty referred or General hospitals in both urban and rural areas in the state was selected based on clustered senatorial zones and stratified random sampling, representing 28 local governments' areas. The case files records of all live deliveries, antenatal attendance, captured maternal death, age at death, parity, educational level and causes of death for all women who died during pregnancy and child delivery between $1^{\text {st }}$ January 2005 and December 31, 2010 were reviewed.

\subsection{Ethical Consideration}

An application was written to the ethical committee through the executive secretary of the Health Management Board ministry of health Kano State for permission to allowed access to information on hospital records of death of pregnant women. All data on cases of delivery, maternal mortality, antenatal attendance, cause of death, age at death and place of residence that occur in all public hospitals and clinics in Kano are recorded by the record and statistics unit attached to maternity wards in all hospitals. Normally all these data are send to each zonal head office responsible for that area which oversees a number of hospitals that are within the zone. Here, data is filter to detect inconsistencies, missing information and other errors in the records submitted from the zone. Once the data is certified here, there are then forwarded to the Research and Statistics Department at the Kano ministry of Health for policy-making purposes. The data presented in this retrospective study is from this source through the permission of ethical committee of the hospital management Board ministry of health Kano State.

\section{Statistical Analysis}

Data was analyzed using Microsoft excel statistical packages and presented in the form of tables, frequencies, percentages, and graphs. The data was also subjected to correlation analysis to establish relationship between the variables.

Data for Maternal Mortality ratio (MMR) was computed by dividing maternal death by the total number of live deliveries multiplied by 100,000 . To know the pattern of maternal mortality ratio for all the years of the study, a complete ratio was calculated for each year and finally an average was taken to arrive at the maternal mortality ratio for the six year period.

\section{Results}

The study reviews the pattern of antennal attendance over six year period for both urban and rural areas in Kano. It indicates that in urban areas there were generally high antenatal attendance compare with rural areas as shown in table1. The highest attendance of $284,823(20.4 \%)$ was found in 2010, while the lowest attendance of 200,000(14.3\%) was found in 2008 for urban area. There was also a decline in 2006, followed by a shaped rise in 2007 but subsequently a steady decline and a raise in 2010 . For rural areas, there was a decline in 2005, a raise in 2006 and a fluctuating increase and decrease and finally a raise of $207,081(22 \%)$ in 2010 . It also shows the pattern of hospital live delivery for urban area higher compared to rural areas. The highest number of 284,122 (20.9\%) deliveries was in 2005 but there was a sharp drop in live deliveries in 2006 and a raise in 2007. In 2010 the number of hospital live deliveries went to 298,304 (22\%) in urban areas. For rural, there was a mixed of up and downs in the number of hospital live deliveries with no clear cut difference. This is in agreement with other studies that look at causes of maternal mortality in northern Nigeria $[5,6,7,8]$. Reasons for such could be due to economic factors and distance to health facilities which influences 
women to deliver at home where relatives can be by their side. It could also be cultural and religious norms or altitudes of family members especially husbands choice for home delivery.

Table 1 also shows a total of 3062 women who died for the study period in government hospitals in Kano, while
1450 and 1612 were for urban and rural areas respectively. The highest number of death for urban and rural areas was in 2010, 312 and 300 deaths respectively. This could be probably due to less attention given to awareness campaign and medical care by the government authorities because of the political year.

Table 1. Shows the record of women participation in obstetric health services in Kano State 2005-2010

\begin{tabular}{|c|c|c|c|c|c|c|c|}
\hline \multirow[t]{3}{*}{ Year } & \multicolumn{2}{|c|}{ Antenatal Attendance } & \multicolumn{2}{|c|}{ Hospital Live Delivery } & \multicolumn{2}{|c|}{ Captured Maternal Death } & \multirow[t]{3}{*}{ MMR } \\
\hline & Urban & Rural & Urban & Rural & Urban & Rural & \\
\hline & $\mathrm{f} \quad \%$ & $\mathrm{f} \quad \%$ & $\mathrm{f} \quad \%$ & $\mathrm{f} \quad \%$ & $\mathrm{f} \quad \%$ & $\mathrm{f} \quad \%$ & \\
\hline 2005 & $218,823(15.7)$ & $114,200(12.5)$ & $284,122(22.6)$ & $97,480(18.4)$ & $300(20.6)$ & 318 (19.7) & 1600 \\
\hline 2006 & $212,321(15.2)$ & $159,919(17.5)$ & $162,301(12.9)$ & 99,511 (18.7) & $240(16.6)$ & $213(13.2)$ & 1768 \\
\hline 2007 & $270,201(19.3)$ & $112,211(12.3)$ & 242,192 (19.3) & $70,580(13.2)$ & 230 (15.9) & $282(17.5)$ & 1637 \\
\hline 2008 & $200,000(14.3)$ & $142,000(15.6)$ & $184,304(14.7)$ & $98,412(18.6)$ & $168(11.6)$ & 307 (19.0) & 1645 \\
\hline 2009 & $212,230(15.2)$ & $177,220(19.4)$ & $185,304(14.8)$ & $73,114(13.6)$ & $200(13.8)$ & 192 (11.9) & 1517 \\
\hline 2010 & $284,521(20.4)$ & $207,081(22.7)$ & 298,304 (16.5.0) & $91,310(17.5)$ & $312(21.5)$ & $300(18.6)$ & 1586 \\
\hline Total & 1,398,096 (100.0) & $912,631(100.0)$ & $1,256,525(100.0)$ & $630,407(100.0)$ & $1450(100.0)$ & $1612(100.0)$ & $\begin{array}{c}9,753 / 6 y r \\
1625\end{array}$ \\
\hline
\end{tabular}

Key: $\mathrm{f}=$ Frequency.

Table 2. Shows the Social -demographic characteristics of women who died during child birth in Kano State 2005-2010 (Number of Captured Maternal Death)

\begin{tabular}{lllll}
\hline Age & Urban & Rural & Total & Percentage (\%) \\
\hline $14-19$ & $170(11.7)$ & $300(18.6)$ & 470 & $(15.3)$ \\
$20-25$ & $238(16.4)$ & $380(23.6)$ & 618 & $(20.3)$ \\
$26-31$ & $312(21.5)$ & $200(12.4)$ & 512 & $(16.7)$ \\
$32-37$ & $300(20.7)$ & $130(8.1)$ & 430 & $(14.0)$ \\
$38-43$ & $230(15.9)$ & $270(16.7)$ & 500 & $(16.4)$ \\
$44-49$ & $200(13.8)$ & $332(20.6)$ & 532 & $(17.3)$ \\
Total & 1450 & 1612 & 3062 & 100
\end{tabular}

\begin{tabular}{lllll}
\hline Parity & & & & \\
& & & \\
\hline $1^{\text {st }}$ delivery & $550(37.9)$ & $700(43.4)$ & 1250 & $(40.8)$ \\
$2-3$ & $600(41.4)$ & $312(19.4)$ & 912 & $(29.8)$ \\
4 \& above & $300(20.7)$ & $600(37.2)$ & 900 & $(29.4)$ \\
Total & 1450 & 1612 & 3062 & \\
\hline Western Education & & & \\
\hline Yes & $412(28.4)$ & $290(18.0)$ & 702 & $(22.9)$ \\
No & $1038(71.6)$ & $1322(82.0)$ & 2360 & $(77.1)$ \\
Total & 1450 & 1612 & 3062 & \\
\hline Booked \& attended ANC & & & \\
\hline Yes & $341(23.5)$ & $64(4)$ & 405 & $(13.2)$ \\
No & $1109(76.5)$ & $1548(96.1)$ & 2657 & $(86.8)$ \\
Total & 1450 & 1612 & 3062 & \\
\hline Source: Computed from hospital data collected at the facility level
\end{tabular}

Source: Computed from hospital data collected at the facility level (2011).

\subsection{Socio Demographic Characteristics of Women Who Died during Pregnancy and Delivery in Kano}

As shown in Table 2, the highest proportion of urban maternal deaths occurred in the 26-31age group with $21.5 \%$ why rural areas had age group of $20-25$ years with $23.6 \%$ as the highest groups that were involved in maternal death during child birth. Therefore the greatest risk of maternal death was among younger women especially in rural areas. Data had shown that death decreases with parity especially in urban areas from 4 and above while rural areas it decreases from 2 to 3 births.
Another important correlate of maternal death was educational level of the women. About $71.6 \%$ and $82 \%$ of all maternal deaths happened in women who had not acquired western education for both urban and rural areas respectively. Literatures have shown that the higher a women level of western education the less likely that she will die a maternal death. Convincingly, the reviewed shows maternal death was more among women who did not participated in antenatal care with $76.5 \%$ in urban areas compared to $96.1 \%$ in rural area. It also shows that women who participated in antenatal care in the hospitals sampled were less involved in maternal mortality with 23.5\% compared to those that did not went through antenatal care.

\subsection{Annual Trends of Maternal Mortality Ratios}

To get a clearer picture of the situation, we now examine the maternal mortality ratio $/ 100,000$ live births. During the period under review a total of 1,398,096 for urban and 912,631 for rural areas participated in antenatal care and 1,256,525 for urban and 630,407 for rural had live deliveries recorded. However, the maternal mortality ratio MMR varied greatly from 1600/100,000 deliveries in 2005 to 1586/100,000 deliveries in 2010. In fact, there was no consistent trend in MMR over the study period; given the highest value of 1762/100,000 live deliveries in 2006 and the lowest MMR of 1586/100,000 live deliveries in 2010 .

Table 3 and Figure 1 show a 6 year trend of Maternal Mortality ratio in Kano State from 2005 to 2010. There was an increase in maternal mortality in 2005 to 2006 by 1762/ 100,000 live deliveries, there after; there was a decrease in maternal mortality in 2007 to 2009 and a slight increase in 2010 to $1571 / 100,000$ live deliveries. A sharp rise in 2006 is an indication of the state of obstetric care services in the state, and it may be lack of information on the importance of antenatal and hospital delivery by the pregnant women and also lack of government participation in health care in terms of better annual budgeting and making sure that the funds allocated to health sector was used appropriately. 
Table 3. Shows the annual trend of maternal mortality ratio in Kano State. 2005-2010

\begin{tabular}{cccc}
\hline Year & Hospital live Deliveries & Maternal Death & MMR/ 100,000 \\
\hline 2005 & 382602 & 612 & 1600 \\
2006 & 262814 & 463 & 1762 \\
2007 & 312772 & 512 & 1637 \\
2008 & 282716 & 465 & 1645 \\
2009 & 258418 & 392 & 1517 \\
2010 & 389614 & 618 & 1586 \\
Total & $1,886,932$ & 3,062 & Average MMR=1625 \\
\hline
\end{tabular}

Source: Computed from hospital data collected at the facility level (2011).

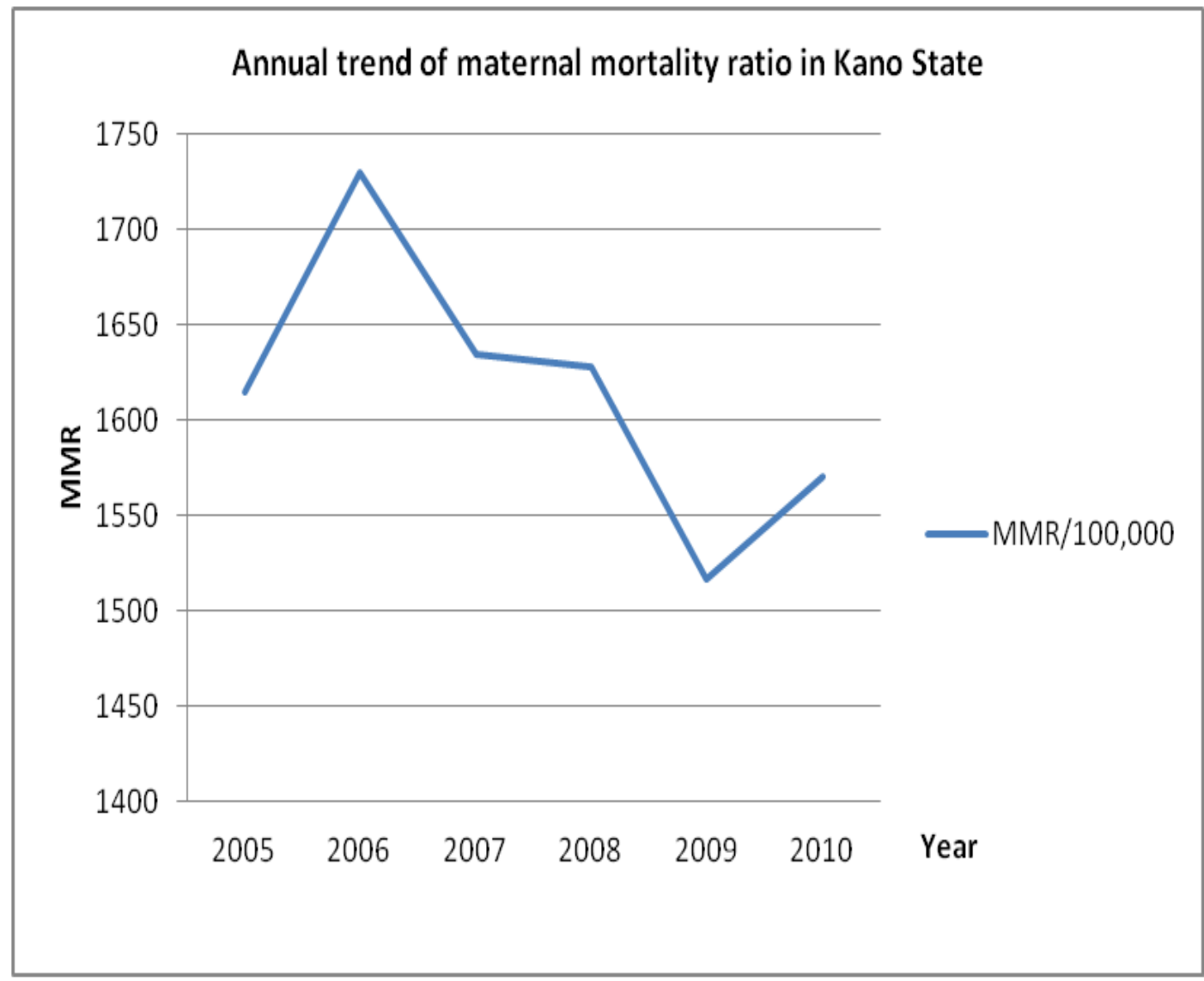

Figure 1. Annual trend of maternal mortality ratio in Kano State

\subsection{Annual Trends of Case Fatality Rates}

Different causes of maternal death were found in the study more especially eclampsia which was $24.3 \%$ for urban and $21.7 \%$ for rural, obstructed labor $6.9 \%$ urban and $18.6 \%$ rural, anemia $3.5 \%$ urban and $12.4 \%$ rural while the direct causes showed $4.1 \%$ for urban and $6.2 \%$ for rural. There was a substantial effect of malaria as a cause of death in the study as shown in Table 4. In order to see the effect of predicted variables on dependent variable a linear regression was run with maternal death as outcome and obstetric complications as the explanatory variable, the result of the analysis gave an R-value of .101 which indicates a positive influence on maternal mortality by the predictor variables. Also, evidently seen was the causes of death between urban and rural areas where the most causes of death in urban area were bleeding cases and eclampsia, in rural areas was a mixed of obstructed labor eclampsia and anemia as a result of early marriage, lack of education and malnutrition.
Table 4. Shows the causes of maternal death for the period under review in Kano State 2005-2010

\begin{tabular}{ccccc}
\hline Causes & \multicolumn{3}{c}{ Number of death from complications } \\
Direct Causes & \multicolumn{2}{c}{ Urban } & \multicolumn{2}{c}{ Rural } \\
\hline & Frequency & Percentage & Frequency & Percentage \\
Bleeding causes & 600 & 41.3 & 400 & $(24.8)$ \\
Eclampsia & 353 & 24.3 & 350 & $(21.7)$ \\
Obstructed labor & 100 & 6.9 & 300 & $(18.6)$ \\
Anemia & 50 & 3.5 & 200 & $(12.4)$ \\
Uterine rupture & 45 & 3.1 & 100 & $(6.2)$ \\
Ectopic pregnancy & 30 & 2.1 & 12 & $(0.7)$ \\
Infection & 200 & 13.8 & 70 & $(4.3)$ \\
Abortion & 120 & 8.3 & 80 & $(4.9)$ \\
Indirect Causes & \multicolumn{5}{c}{100} \\
Malaria and others & 60 & $(4.1)$ & 100 & $(6.2)$ \\
$\quad$ Total & 1450 & $(100.0)$ & 1612 & $(100.0)$ \\
\hline Source: Computed from hospital data collected at the facility level \\
(2011).
\end{tabular}




\section{Discussion of Findings}

This study detected very high maternal mortality ratios (MMR) at the study sites. These values by far exceed the average of 1000 maternal deaths per 100,000 deliveries estimated for Africa; the continent with the highest MMR on the planet [9].It is no exaggeration, therefore, that the average maternal mortality ratio of 1625 per 100,000 deliveries found in this study is one of the highest population-based MMRs reported within this region. When comparison is made with other studies on maternal mortality published in Nigeria within the last decade it is clearly the most disturbing scenario. A probable explanation for this could be the difference in the study design and target population. While data in this study were also hospital-based, almost all the other studies were based on cases of maternal deaths observed among deliveries that took place in teaching hospital settings so inferences deduced might not have been representative of the general population. Patients that deliver in teaching hospitals are likely to be more enlightened with regard to health issues, can afford quality care offered in teaching hospitals, and many of them live in the urban areas so the physical access to ante-natal care and hospital delivery may not constitute a big issue. This is in contrast with the socio-economically disadvantaged mothers living in the remote and neglected rural areas where illiteracy and poverty-reign supreme. The comprehensive and primary health centers are poorly staffed and ill-equipped, and complicated deliveries have to be managed on-site due to physical, social and economic barriers which often preclude referrals to higher levels of care. Moreover, in many cases, such referrals are perceived by patients and their relatives as acts devoid of compassion or acts that simply delay the inevitable death of the mother. Most of these complicated deliveries therefore remain and die at these centers. The maternal deaths in these areas not served by teaching hospitals are therefore, more likely to be higher than the figures reported in previous reports. By being population-based, our study took these neglected maternal deaths into account in order to obtain less biased estimates.

There was an increase trend in antenatal attendance for both urban and rural areas, with a substantial number of hospital deliveries for urban area. Unfortunately, hospital deliveries for rural area were marked with annual fluctuations of not improved figures consistent with the findings from other studies [1,9,10,11]; also the rural areas had the highest maternal deaths of 1612 deaths/ 100,000 lives deliveries during the period of the study. This is because girls are married out early in rural areas when their not mature for the rigors of pregnancy and delivery and uneducated as their urban counterparts to know the danger signs of pregnancy.

There was no consistent pattern of antenatal attendance or hospital deliveries for both urban and rural areas during the 6- year study. The figures only reflect the differences in antenatal attendance between urban and rural areas showing more improved figures for urban area than rural area. The reason for such is because urban pregnant women tend to benefit from increase knowledge and access to maternal health services compared with their rural counterparts, also health facilities are more accessible in urban areas and the various health promotion programs that use urban- focused mass media work to the advantage of urban residents. Moreover, rural pregnant women are more readily influenced by traditional practices that are contrary to the modern health care. Other reasons affecting rural women include inability to obtain transportation in time, distance to health facilities, poverty and low status accorded to women especially rural women and lack of education [1,12].

The maternal mortality ratio (MMR) found in the study is not a conclusive result considering the fact that substantial number of maternal deaths are unaccounted for as the result of deliveries taking place at home and private hospitals in Kano. This means the analysis is not a true representative of the true situation on the ground. In addition, the difference in MMR between urban and rural areas could be because of the difference in illiteracy level of the two groups. Illiteracy is found to be closely associated with maternal mortality ratio, the findings is in agreement with previous studies in Nigeria $[15,16,17,18]$. Education is likely to enhance female autonomy so that women develop greater confidence and capability to make decisions about their own health [1]. It is also likely that educated women seek out higher quality services and have greater ability to use health care inputs that offer better care. The pattern of maternal mortality ratios recorded in this study indicate that maternal mortality in Kano State is a serious problem if more immediate interventions are not given to poverty, health education, improvement in social amenities and obstetric services. Inconsistent and ever changing health policies by different governments coupled with insecurity, lack of awareness or information on the best practices by pregnant women, negligence by our political class and socio- cultural factors may have contributed to this fluctuating MMR found in this study.

It is interesting to note the epidemic pattern of maternal mortality ratios within the 6 year study period, which demonstrates that when population data on maternal deaths are employed to describe the occurrence of these events a lot more useful information could be obtained than the popular use of a descriptive average, which may convey the wrong impression of a "static equilibrium." Our data clearly shows that maternal mortality occurrence in developing areas of the world, such as Nigeria, could exhibit episodes of outbreaks similar to the phenomenon of infectious disease epidemics as seen in rural areas where young girls are married out at a young age of 10-12 years depriving them of their rights to health and western education subsequently compounding their social and medical life. It is therefore important that we consider maternal mortality occurrence as "dynamic process" associated with triggering factors. Unidentified triggers of maternal death epidemics are most likely also the root causes of the unstable baseline values of maternal deaths. Given this concept, it becomes clear that one of the most effective strategies in understanding and consequently curtailing the high maternal mortality ratios in developing countries, especially, sub-Saharan Africa and Nigeria in particular is the establishment of a sustainable "Surveillance System" that constantly monitors this dynamic occurrence of maternal mortality in the population.

\section{Conclusion}

The study found that there was an increased trend in antenatal attendance but slight increase in hospital 
deliveries more pronounce in urban areas than rural areas. The number of hospital deliveries was far less than the number of antenatal attendance more especially in rural areas.

The MMR in Kano (North western Nigeria) has remained high but the trend is gradually decreasing. The difference between urban and rural areas is distinct because of several factors that play in the lives of this subgroup. The highest cause of death is found to be bleeding disorders and eclampsia generally, but the difference was seen within the groups. For example, in urban areas bleeding and eclampsia disorders were the main causes of death, whereas, in rural areas eclampsia, obstructed labor and bleeding causes future prominently as causes of death. There is the disparity in age at marriage between urban and rural settings. Age, western education was found to be a determinant factor of maternal mortality in the study area leading to prolonged obstructed labor mostly resulting to death during delivery. Death was also seen to decrease with parity especially in four and above in urban areas and two to three in rural areas in the study.

\section{Limitations and Implications for Further Research}

This study did not take into consideration the fact that some women that died were not due to complications arising from none obstetric causes in the records of facilities examined also some may died because of indirect causes of maternal mortality in which the data was not specific in addressing such concerns. Further studies should take these short comings into account. Apart from these recorded deaths, there was a possibility that some of the pregnant women might have died at home or private hospitals whose cases are not reported here. For this reason, the maternal mortality ratio for Kano State might probably be more than what is calculated in this study therefore it can not be generalized. Despite all these challenges, the data received from government hospitals have given us some insight into the proportion of those women who died among those who had live births in Kano State. Furthermore, it will be important in future studies to look at why death was found to be decreasing when parity increases and also to include death from private hospitals and death outside hospitals so as to arrive at the more accurate figures of maternal mortality in Kano State.

\section{Conflict of Interests}

None declared.

\section{Funding}

The authors would like to acknowledge the charitable trust monies from professor Gateel foundation which funded the data collection and analyses of the research.

\section{References}

[1] World Health Organization (WHO) Maternal Mortality in 2007: Estimates Developed by WHO, UNICEF, UNFPA, and the World Bank, 2006; Geneva.

[2] Advocacy Brief, Integrated Approach to Improve Maternal, Newborn and Child Health Action Points for the Media. Produced by the Federal Ministry of Health with support from ENHANCE project/USAID. 2007.

[3] NDHS, Assessment of DHS Maternal Mortality Indicators Studies in Family Planning, 2008; 31: 111-23.

[4] National Population Commission (NPC) [Nigeria] and ORC Macro. Nigeria Demographic and Health Survey 2008. Calverton, Maryland: National Population Commission and ORC Macro.

[5] WHO, "Maternal Mortality in 2008: Estimates Developed by WHO/UNICEF/UNFPA." WHO, 2007; Geneva.

[6] Ejembi CL, Alti- Muazu M, Chirdan O, Ezeh HO, Sheidu S. Utilization of maternal health services by rural Hausa women in Zaria environs, northern Nigeria: has primary health care made a difference? Journal of Community Medicine and Primary Health Care.2004; 16 (2): 47-54.

[7] Audu LR, Ekele BA, A ten year review of maternal mortality in Sokoto Northern Nigeria. West Afr. J med. 2002; 21: 74-6.

[8] Dan Yaro, M.M. Ministry of information, Youth and culture bulletine, Kano.2010; 22, 4-8.

[9] Adamu M Yusuf, Hamisu M Salihu, Nalini Sathiakumar, Maternal mortality in northern Nigeria: a population-based study. Euro J Obstet Gynecol Reprod Biol. 2003; 109(2): 153-159.

[10] Harrison K., Maternal Mortality in Nigeria: The Real Issues. African Journal of Reproductive Health.1997; 1(1): 7-13.

[11] Ujah IA, Aisien OA, Mutihir JT, Vanderjagt DJ, Glew RH, Uguru VE, Maternal mortality among adolescent women in Jos, North Central, Nigeria. J Obstet Gynaecol; 2005; 25: 3-6.

[12] Galadanci, H., Kunzel, W., Shitu, O., et al, Obstetric quality assurance to reduce maternal and fetal mortality in Kano and Kaduna States, Nigeria. Int. J. of Gynecology and Obstetrics. 2011; 114, 23-28.

[13] Abuser O, The Millennium Development Goals: can Nigeria meet the goals in 2015? paper presented at the Symposium on Millennium Development Goals and Nigeria: Issues, Challenges and Prospects, Abuja, Nigeria,

$<$ http://www.civicus.org/new/media/ accessed Sep. 19, 2008. 2006; 1(1): 7-13.

[14] Stanton C., Hobcraft J., Hill K., Kodjogbe N., Mapeta W. T.,(2001) Every Death Counts: Measurement of Maternal Mortality Via Census. Bulletin of the World Health Organization. 79(7): 657-64.

[15] Adetoro A.A, Taiwo OL, Martins OO,Ann MT, A community based investigation of the avoidable factors of maternal mortality in Nigeria: The pilot experience. Afr Health sc, 2007; 7: 176-81.

[16] Lambo E, Breaking the cycle of poverty, ill-health and underdevelopment in Nigeria, paper presented at a special Guest Lecture of the College of Medical Sciences, University of Benin, 2006; Benin City, Nigeria.

[17] FMOH, Advocacy Kit: Improving maternal, newborn and child health, Federal Ministry of Health (2001) National Reproductive Health Policy and Strategy. 2008; Abuja, Nigeria.

[18] Chiwuzie, J. and Okolocha C, "Traditional belief systems and Maternal Mortality in a Semi Urban Community in Southern Nigeria". African Journal of Reproductive Health, 2001; 5 (1), 7582.

[19] WHO, UNICEF, UNFPA. Advancing Safe Motherhood through Human Rights. Available

http://www.who.int/reproductivehealth/publications/RHR_2010;a dvancing safe motherhood HR_01_05_table_of_contents_en.html. 